\title{
Protective effect of sensory denervation in inflammatory arthritis (evidence of regulatory neuroimmune pathways in the arthritic joint)
}

\author{
D Kane, J C Lockhart, P V Balint, C Mann, W R Ferrell, I B Mclnnes

Objective: To investigate the direct effect of joint innervation on immune mediated joint inflammation in a patient with psoriatic arthritis (PsA).

Case report: The patient developed arthritis mutilans in all digits of both hands with the exception of the left 4th finger, which had prior sensory denervation following traumatic nerve dissection. Plain radiography, ultrasonography and nerve conduction studies of the hands confirmed the absence of articular disease and sensory innervation in the left 4 th digit.

Methods: This relationship between joint innervation and joint inflammation was investigated experimentally by prior surgical sensory denervation of the medial aspect of the knee in six Wistar rats in which carrageenan induced arthritis was subsequently induced. Prior sensory denervation-with preservation of muscle function - prevented the development of inflammatory arthritis in the denervated knee.

Discussion: Observations in human and animal inflammatory arthritis suggest that regulatory neuroimmune pathways in the joint are an important mechanism that modulates the clinical expression of inflammatory arthritis.

A 60 year old woman presented with psoriasis, which she had had since age 16, and psoriatic arthritis (PsA) since age 45. Initial involvement of the right hand had progressed to an inflammatory polyarthritis, affecting the small joints of both hands and both knees, and to dactylitis of the toes but without spondylitis or sacroiliitis. Current drug treatment comprised methotrexate and rofecoxib and there were no signs of active synovitis of the hands, either upon clinical examination or by laser Doppler imaging.

On examination the patient was noted to have established arthritis mutilans of the metacarpophalangeal (MCP), proximal interphalangeal (PIP), and distal interphalangeal (DIP) joints of the hands with complete sparing of the left 4 th digit (fig 1A). Of note the left palm had been lacerated as a child-before the onset of skin or joint disease-with consequent nerve injury and loss of sensation in the left 4 th finger. The left 4 th digit was noted to be hypoplastic with a normal range of active and passive movement but with loss of soft touch, pinprick, and vibration sensation along the entire digit distal to the laceration. Investigations were consistent with inactive PsA (rheumatoid factor negative, C reactive protein $<6 \mathrm{mg} / \mathrm{l}$, erythrocyte sedimentation rate $1 \mathrm{~mm} / \mathrm{lst} \mathrm{h}$ ).

Plain radiography of the hand confirmed a symmetrical destructive arthropathy of the MCP, PIP, and DIP joints with features of bony resorption and periosteal proliferation in all digits, but with sparing of the small joints of the left 4 th digit (fig 1B). Ultrasonography-a more sensitive measure of joint inflammation and damage - of PIP joints in the right and left 4th digits demonstrated proliferative changes in the right 4th digit but not in the left 4 th digit (fig $1 \mathrm{C}$ ). Nerve conduction studies confirmed the complete absence of sensory innervation in the left 4 th digit. On stimulation of the 4th digit no reproducible sensory responses could be obtained over either the median or ulnar nerves on the left side while normal responses were obtained from both the median and ulnar nerves on the right (fig 1D).

\section{Denervation animal studies}

Division of the saphenous nerve in the hind limb of the rat results in partial sensory denervation of the knee joint but does not immobilise the joint or lead to motor deficits. Synovial inflammatory hyperaemia was quantified using transcutaneous laser Doppler imaging (Lisca, Sweden) to assess synovial blood flow. ${ }^{2}$ The sensitivity of laser Doppler in assessing synovial perfusion transcutaneously has been previously confirmed in both animal ${ }^{2}$ and human joints. ${ }^{1}$ The depilated intact knee joints of deeply anaesthetised (Sigma UK; $1.6 \mathrm{~g} / \mathrm{kg}$ urethane intraperitoneally) male Wistar rats $(\mathrm{n}=4)$ were scanned after "sham denervation" ( surgical skin incision but nerve left intact) and intra-articular injection of $2 \% \lambda$-carrageenan and $4 \%$ kaolin (wt/vol; Sigma). The increased colouration of the left hand panels in fig 2A demonstrates the developing inflammatory hyperaemia over 6 hours, a vascular response notably absent in a parallel group of rats $(n=6)$ in which the medial aspect of the knee joint had been surgically denervated by resection of the proximal saphenous nerve 1 week earlier (fig 2A: right hand panels). Furthermore, the substantial knee joint swelling (assessed using callipers) evident in sham operated animals (figs 2B, C) was greatly attenuated in denervated knees (fig 2B, C).

\section{DISCUSSION}

Although it is possible that the clinical findings in this patient with PsA are coincidental, a number of reports suggest otherwise. A patient with a longstanding hemiplegia who subsequently developed psoriasis and arthritis on the non-hemiplegic side but not the hemiplegic side has been reported. ${ }^{3}$ The mechanism of joint sparing was postulated to have been related to either sensorimotor denervation of the hemiplegic side or to a lack of joint movement and stressing subsequent to the hemiplegia. In a second case, sparing of DIP joint disease and nail dystrophy in a partially denervated digit was reported in a patient with chronic PsA. ${ }^{4}$ In this case, clinical examination confirmed that the joint was moving

Abbreviations: DIP, distal interphalangeal; MCP, metacarpophalangeal; PIP, proximal interphalangeal; PsA, psoriatic arthritis 


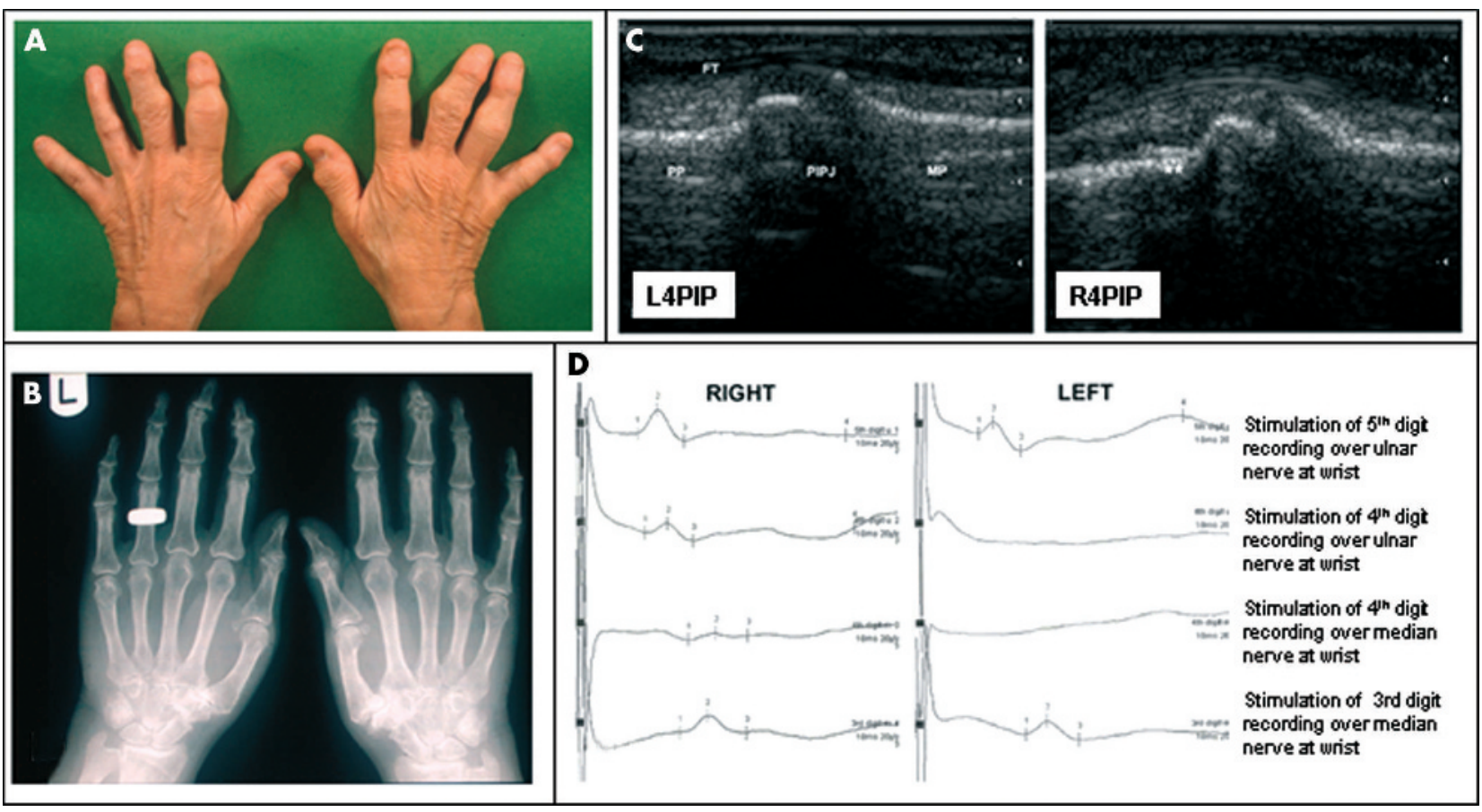

Figure 1 (A) Swelling and deformity affecting most of the DIP and PIP joints of both hands. The left 4 th digit is hypoplastic and has no evidence of joint inflammation or deformity. (B) Plain radiography demonstrates typical erosive joint damage and periostitis of PsA. The changes are most marked in the DIP joints and to a lesser extent in the PIP joints. (C) High resolution ultrasound of the left 4th PIP joint shows the clear bony outlines of the palmar aspects of the proximal phalanx (PP), PIP joint (PIPJ), and middle phalanx (MP) with the flexor tendon (FT) overlying. On the right, bony proliferation is present proximal (**) and distal to the PIP 4th joint. (D) Orthodromic nerve conduction studies from the right and left hands. From both hands stimulation of the 5 th digit produced a normal ulnar sensory response. Stimulation of the right 4 th digit produced a normal ulnar and median response. Stimulation of the left 4 th digit produced no ulnar or median sensory response. In both hands stimulation of the 3rd digit produced a normal median sensory response. All waveforms were reproducible.

normally and that there was partial sensory denervation affecting a small area on the lateral and distal aspect of the digit. In our patient's case the nerve supply to the left 4 th digit was further detailed using nerve conduction studies that confirmed the complete absence of sensory conduction with normal motor function of the digit. Taken together these studies suggest that sensory denervation-and not joint immobility after motor nerve damage-inhibits the expression of inflammatory joint disease in PsA.

Animal and human studies provide further evidence of the influence of sensory innervation on synovial inflammation. Human rheumatoid arthritis synovium expresses markers of increased sensory innervation (substance P expression) and decreased sympathetic innervation (tyrosine hydroxylase expression) in comparison with osteoarthritic synovium. ${ }^{5}$ Whether these changes develop before or after the onset of synovial inflammation is not known, though studies of synovial innervation in the adjuvant arthritis model suggest that increased synovial nerve fibre activation and degeneration occurs after the onset of arthritis. ${ }^{6}$ In the case of the patient with PsA and hemiplegia, synovial fluid levels of substance $\mathrm{P}$ were reduced in the knee on the hemiplegic side despite abundant synovial membrane substance P expression in both knees. One interpretation of this is that substance P release by sensory nerves in the synovium is inhibited by denervation, and substance $\mathrm{P}$ release may be an important factor in promoting the clinical expression of inflammatory arthritis in PsA.

Capsaicin-trans-8-methyl- $N$-vanillyl-6-nonenamidedepletes substance $\mathrm{P}$ in small unmyelinated type $\mathrm{C}$ sensory neurones, and systemic administration of capsaicin in the guinea pig and rat models of adjuvant arthritis produces partial generalised sensory denervation and attenuation-but not prevention - of macroscopic joint inflammation, radiological damage, and synovial infiltration by $\mathrm{T}$ lymphocytes. ${ }^{7}$ However, systemic administration of capsaicin does not selectively target articular nerves, and anti-inflammatory and other toxic effects may be mediated by the action of capsaicin at non-articular sites. As the development of PsA had been prevented in our patient by sensory denervation we proceeded to test this hypothesis by investigating the effect of localised surgical selective sensory denervation of the knee in an animal model of acute inflammatory arthritis. This confirmed that prior sensory denervation attenuated acute joint inflammation-known to be mediated via complement activation of the alternate pathway-in the animal model of carrageenan induced arthritis.

This report highlights a mechanism whereby clinical expression of PsA-a chronic autoimmune disorder-is attenuated by sensory denervation of the articular tissues. Furthermore, prior sensory denervation in the carrageenan induced arthritis model prevents acute inflammation. The relationship between the expression of synovial inflammation and synovial innervation is highly complex and a number of nerve growth factors and neuropeptides are up regulated in the inflamed synovium. ${ }^{8}$ Surgical sensory denervation is a non-specific method of reducing the release of these factors in the joint and clearly could not constitute an acceptable form of anti-inflammatory treatment. However, this report provides evidence that the elucidation and specific modulation of intra-articular innervation and neuropeptide release represents a new and unused therapeutic approach in suppressing joint inflammation in PsA. 

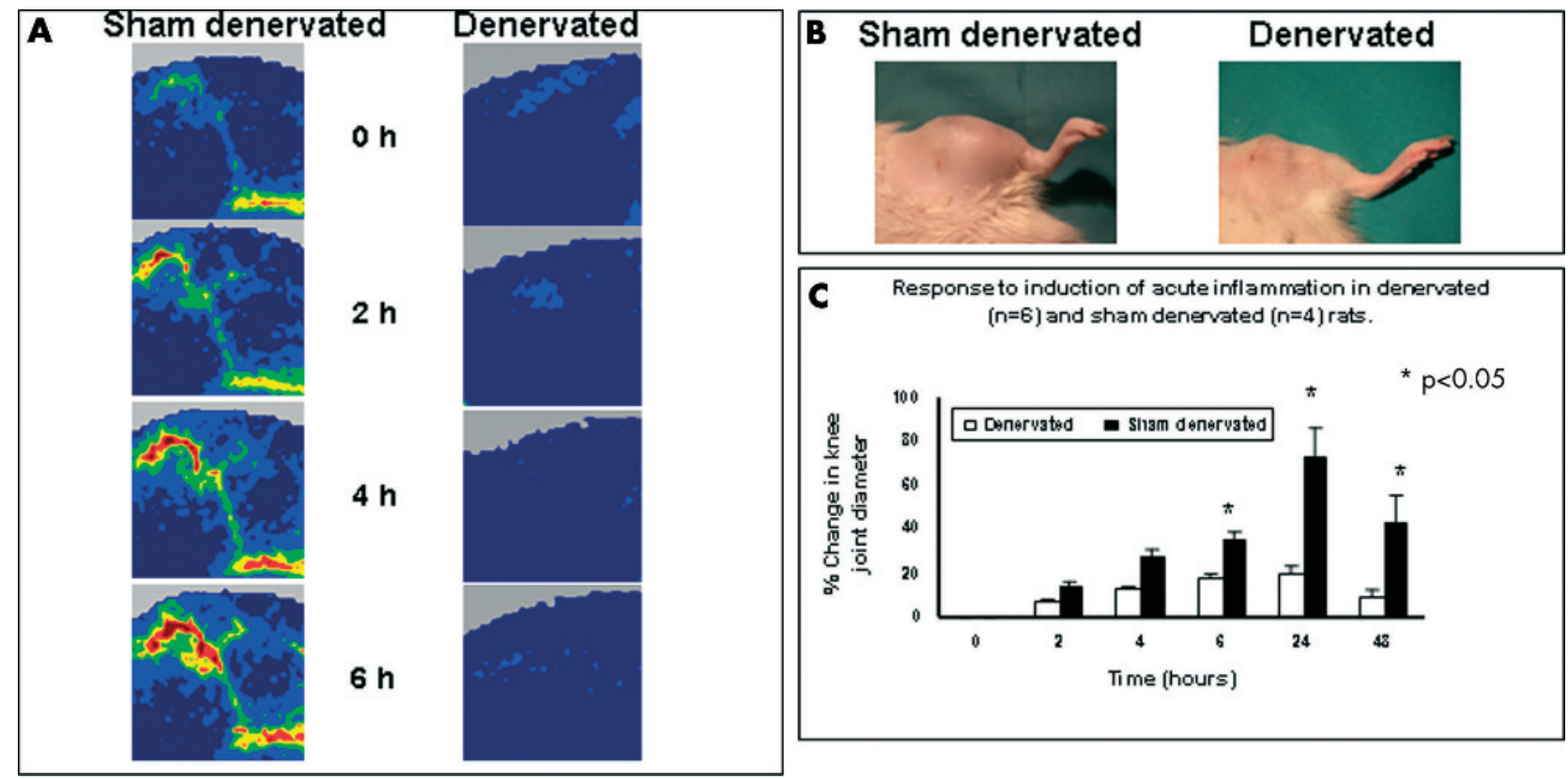

C Response to induction of acute inflammation in denervated $(n=6)$ and sham denervated $(n=4)$ rats.

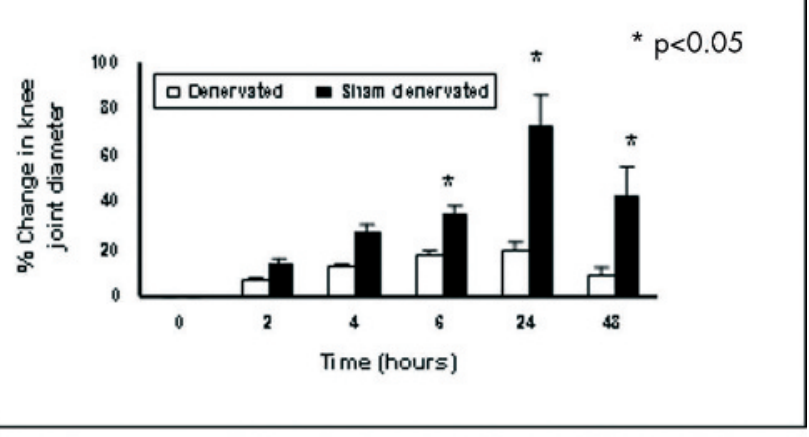

Figure 2 (A) Perfusion images obtained from depilated rat knee joints scanned transcutaneously using a near-infrared (830 nm) laser Doppler imager. Acute inflammation was induced by injection of carrageenan/kaolin in both sham operated and denervated joints. Perfusion (flux) is colour coded with the lowest perfusion in dark blue, and the highest in red. Inflammatory hyperaemia fails to develop in the denervated knee. (B) At 24 hours after intra-articular carrageenan injection there is marked soft tissue swelling of the knee joint of the sham denervated animal but not in the denervated animal. (C) Changes in diameter of the knee joint over 48 hours demonstrate a rapid increase in swelling of the knee joint in the sham denervated animals, maximal at 24 hours, compared with the minimal change in joint diameter in the denervated animals. Student's $t$ test with Bonferroni correction.

\section{Authors' affiliations}

D Kane, P V Balint, W R Ferrell, I B Mclnnes, Centre for Rheumatic Diseases, University Department of Medicine, Glasgow Royal Infirmary, UK

J C Lockhart, Biological Sciences, University of Paisley, UK

C Mann, Institute of Neurological Sciences, Southern General Hospital, Glasgow, UK

Correspondence to: Dr D Kane, School of Clinical and Medical Sciences (Rheumatology), 4th Floor, Cookson Building, Framlington Place, University of Newcastle-upon-Tyne, Newcastle-upon-Tyne NE2 4HH, UK; d.j.kane@ncl.ac.uk

Accepted 7 April 2004

Published Online First 21 May 2004

\section{REFERENCES}

1 Ferrell WR, Balint PV, Egan CG, Lockhart JC, Sturrock RD.

Metacarpophalangeal joints in rheumatoid arthritis: laser Doppler imaging initial experience. Radiology $2001 ; 220: 257-62$.
2 Ferrell WR, Lockhart JC, Karimian SM. Tachykinin regulation of basal synovial blood flow. Br J Pharmacol 1997; 121:29-34.

3 Veale $D$, Farrell M, Fitzgerald $O$. Mechanism of joint sparing in a patient with unilateral psoriatic arthritis and a longstanding hemiplegia. $\mathrm{Br} J$ Rheumatol 1993:32:413-16.

4 Mulherin D, Bresnihan B, FitzGerald O. Digital denervation associated with absence of nail and distal interphalangeal joint involvement in psoriatic arthritis. J Rheumatol 1995;22:1211-12.

5 Miller LE, Justen HP, Scholmerich J, Straub RH. The loss of sympathetic nerve fibers in the synovial tissue of patients with rheumatoid arthritis is accompanied by increased norepinephrine release from synovial macrophages. FASEB J 2000;14:2097-107.

6 Imai S, Tokunaga Y, Konttinen YT, Maeda T, Hukuda S, Santavirta S. Ultrastructure of the synovial sensory peptidergic fibers is distinctively altered in different phases of adjuvant induced arthritis in rats: ultramorphological characterization combined with morphometric and immunohistochemical study for substance $P$, calcitonin gene related peptide, and protein gene product 9.5. J Rheumatol 1997;24:2177-87.

7 Hood VC, Cruwys SC, Urban L, Kidd BL. The neurogenic contribution to synovial leucocyte infiltration and other outcome measures in a guinea pig model of arthritis. Neurosci Lett 2001;299:201-4.

8 Konttinen YT, Kemppinen P, Segerberg M, Hukkanen M, Rees R, Santavirta S, et al. Peripheral and spinal neural mechanisms in arthritis, with particular reference to treatment of inflammation and pain. Arthritis Rheum 1994;37:965-82. 